\title{
Fungal food spoilage of supermarkets' displayed fruits
}

\author{
Iman Saleh and Roda Al-Thani \\ Department of Biological and Environmental Sciences, College of Arts and Sciences, Qatar University, P.O. Box 2713, \\ Doha, Qatar. \\ Corresponding author: Iman Saleh, e-mail: imanesaleh@qu.edu.qa \\ Co-author: RA: ralthani@qu.edu.qa \\ Received: 08-07-2019, Accepted: 22-10-2019, Published online: 29-11-2019
}

doi: www.doi.org/10.14202/vetworld.2019.1877-1883 How to cite this article: Saleh I, Al-Thani R (2019) Fungal food spoilage of supermarkets' displayed fruits, Veterinary World, 12(11):1877-1883.

\begin{abstract}
Background and Aim: Post-harvest fungal infection of fruits and vegetables is mainly caused by fungal pathogens that can be harmful to both human and animals as they produce mycotoxins, post-harvest diseases in fruits and vegetables are a serious problem that results in the loss of a large percentage of crops reaching $50 \%$ in some fruits. This study aims at screening the post-purchasing shelf-life of four highly consumed fruits and vegetables and at identifying the fungal strains behind their spoilage in Qatar.
\end{abstract}

Materials and Methods: Fruits and vegetables were collected from the market to study their post-purchasing shelf-life and to identify the fungal types involved in samples rotting. Factors that affect samples' shelf-life were also analyzed.

Results: A total of 73 fungal isolates were isolated and identified, with the highest percentage of Penicillium (21.9\%) followed by Rhizopus (17.8\%). Interestingly, many mycotoxins producing and diseases inducing fungi were identified in this study; this includes Rhizopus, Aspergillus, Penicillium, Alternaria, Fusarium, Cladosporium, Botrytis, Geotrichum, and Colletotrichum. Statistical analysis shows that different fruits have significantly different shelf-life and different predispositions for spoilage. In many cases, a strong relationship was shown between the fungal types isolated and the country of origin of the fruit. Finally, the price of the commodity did not have a significant effect on its contamination level nor did the market from which the sample was purchased. This indicates that the fruit displaying methods in Qatar do not affect their contamination level.

Conclusion: The study is among the first reports about fungal types involved in fruits and vegetables rotting in Qatar and it highlights the strong link between spoiling fungi and their country of origin.

Keywords: country of origin, fungi, post-harvest, shelf-life, spoilage.

\section{Introduction}

Microbiological food safety is a major economic and public health concern nowadays. According to the WHO, one in every 10 people become ill from consuming contaminated food each year, a trend that results in the death of 420,000 individuals annually WHO [1]. Food contaminants consist of physical agents, including pieces of metals or plastic, that enter food during the packaging stage of manufacture, as well as other chemical agents including heavy metals, pesticides, and, most importantly, microbes [2,3].

Fruits are a major source of nutrients for humans and animals, but it has been reported that, globally, around $45 \%$ of harvested fruits and vegetables are wasted every year due to spoilage caused by contaminated growth environments, inappropriate harvesting conditions, unsafe handling and storage processes, and incorrect methods of display [3]. Even though freshly harvested vegetables and fruits are unavoidably contaminated with

Copyright: Saleh and Al-Thani. Open Access. This article is distributed under the terms of the Creative Commons Attribution 4.0 International License (http://creativecommons.org/licenses/ by/4.0/), which permits unrestricted use, distribution, and reproduction in any medium, provided you give appropriate credit to the original author(s) and the source, provide a link to the Creative Commons license, and indicate if changes were made. The Creative Commons Public Domain Dedication waiver (http:// creativecommons.org/publicdomain/zero/1.0/) applies to the data made available in this article, unless otherwise stated. a variety of bacteria, fungi and other microorganisms. However, molds in general and mycotoxin producers in particular are the main cause of spoilage, especially in products that are refrigerated in open boxes [4-6].

Microorganisms, including bacteria and fungi, cause considerable economic losses by spoiling not only harvested fruits and vegetables but also crops in their fields. The identification of such spoilage microorganisms is a crucial step toward controlling them. Some pathogenic strains specific to fruits are pathogenic to humans as well, especially those that produce toxins $[5,7]$. The metabolites of many such microorganisms are heat stable, which suggests that they remain in the food after heat processing and continue to cause toxicity. Once mycotoxins are formed, it is difficult to manage their quantities as they are stable under storage conditions and particularly insensitive to physical and chemical treatments. Therefore, the best way to limit mycotoxin exposure is to stop them from forming in the first place $[6,8,9]$.

Different spoilage-causing microorganisms have different nutrients requirements. Due to the variable composition of fruits and vegetables, it is important to determine the microbial hazards for each product separately. In Qatar, the country imports over $90 \%$ of its consumed food. Food security as one of the major challenges of the modern world is mentioned as one 
of the pillars of Qatar's National Vision 2030, which places great importance on fungal contamination control. Products of great concern include cucumbers and tomatoes, which are produced in large amounts in Qatar and are among the most highly consumed vegetables in the Middle East, as well as oranges and strawberries, which are used in great quantities for the production of fresh juices. Therefore, it is of significant concern to understand the spoiling agents behind their shelf-life termination $[10,11]$.

In this study, samples of cucumber, tomato, strawberry, and orange were tested to determine their shelf life and to identify spoiling agents, if found. Factors that affect the rate of fungal spoilage in the purchased samples were also analyzed.

\section{Materials and Methods}

\section{Ethical approval}

This study did not involve the use of live animals, and hence, ethical approval was not required.

\section{Sample collection}

Samples were collected during four trips which took place between September 2017 and November 2017. All trips occurred on Saturday and samples were collected between 10.00 am and $12.00 \mathrm{pm}$ from three large supermarkets in Qatar. At each market, a sample was collected per available type of cucumber, tomato, orange, and strawberry. Samples were handled aseptically, kept in sterile bags with a breathable patch $44 \mathrm{~cm} \times 20.5 \mathrm{~cm}$ (Sun bag, transparent, SIGMAALDRICH, Montreal, QC), and were assigned serial numbers. All necessary data regarding each sample were collected including the product's country of origin, its type, and the price per kg. Samples were kept in the sterile bags and at a storage temperature approximating that of the supermarket until transferred to the laboratory the next day.

\section{Sample processing}

The sample collection was kept individually in a sterile and empty Petri dish with a sample number identifier. All samples were incubated at $25^{\circ} \mathrm{C}$ and observed twice a day to determine when each would rot. When a sample began to exhibit fungal hyphae growth, it was removed from the incubator, at which point, the observer noted the day as the end of the home shelf-life of that particular sample. Hyphae were subsequently collected using a sterilized needle and subcultures on potato dextrose agar (PDA) [12]. The number of spoiling spots on each sample was counted as a measure of contamination level. If more than spoiling spot color and shape appear, each spot's hyphae were cultured separately. Rotten fruits were discarded in hazardous waste and the inoculated PDA plates were separately incubated at $25^{\circ} \mathrm{C}$. The process continued for 10 days, after which time, the experiment was completed.

\section{Identification of isolated fungi}

After 1 week of incubation, fungal isolates were identified using colonies and cell morphological features such as the thallus growth pattern, pigmentation, conidiophore, and conidial morphology [13]. Isolated fungi were identified using cotton blue in lactophenol stain. A drop of the stain was placed on a clean slide and a portion of the mycelia was placed on the stain using a sterilized needle and forceps. A coverslip was then placed on the wet mount and the slide was examined by a light microscope at various magnifications. The morphology and characteristics of the conidia and conidiophores were then used to classify the different types of fungus according to the standard taxonomic system [5]. Data were entered into the excel datasheet, pictures were taken of the fungi on the PDA agar, and pictures of each isolate under the microscope showing conidia and conidiophores were taken.

\section{Statistical analysis}

Data were analyzed using SPSS statistical software, version 24 (IBM Corp., NY, USA). Chi-square test and ANOVA were applied against an acceptance limit of $p<0.05$. Chi-square was used to determine if there were significant differences in the detection of spoiling among the four samples type, the three collection markets, the eight countries of origin, and the two levels of the countries' economic conditions and commodity price.

\section{Results}

\section{Fungal isolates}

Out of the 90 samples, 51 (56.7\%) showed fungal growth within the experiment timeline (10 days). Various types of fungus were isolated from different fruits. Table-1 shows the number of various fungal types isolated from each kind of fruit. A total of 73 fungal isolates were collected and identified, with the highest percentage of Penicillium (21.9\%) followed by Rhizopus (17.8\%). Different mycotoxins producing fungi were isolated in the study.

\section{Samples contamination rates}

Chi-square test was used to detect the factors that significantly affect fruit spoilage: Sample type, the market from which the samples were purchased, and the sample's country of origin. Our results show that $100 \%$ of the collected strawberry samples became rotten within the 10 day experimental timeframe followed by $81.25 \%$ of the cucumber samples. On the other hand, $60 \%$ of the tomato samples became rotten during the experiment, while only $20 \%$ of the oranges showed fungal growth within the same time period. The results of the Chi-square test indicate that the fungal-driven spoiling rates of the various fruits are significantly different $(\mathrm{p}=0<0.01)$.

\section{Effect of displaying market on fruit spoilage}

Samples were collected from three large supermarkets in Doha. A Chi-square test demonstrated no significant difference among the different markets in terms of the number of samples showing fungal spoilage within 10 days $(\mathrm{p}=0.87>0.05)$, which implies that 


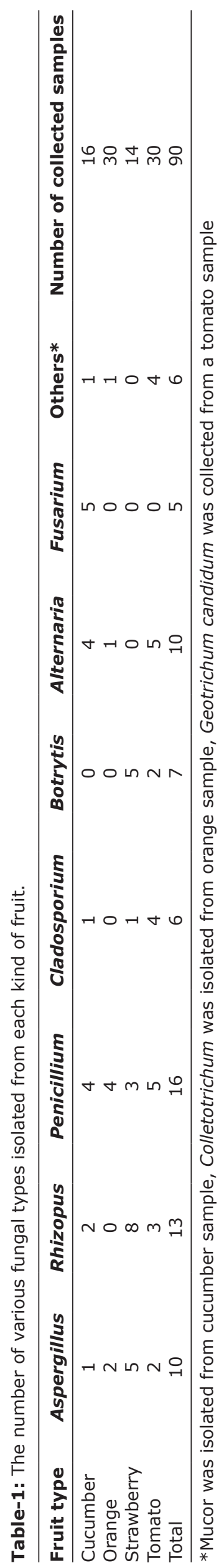

contamination is country of origin related and not induced in market.

\section{Effect of fruits country of origin on fruit spoilage}

When testing for the effect of the country of origin on commodities' fungal spoilage, the Chi-square $\mathrm{p}$-value obtained was $0.02(\mathrm{p}<0.05)$; however, the Chi-square assumptions in this case were not valid (10 cells have expected count $<5 \%$ ). Chi-square test results of the comparison of the number of samples showing fungal growth among samples originated from different countries suggest that the country of origin has an effect on the contamination of commodities, although significance could not be proven, as more samples were needed to reach an adequate level of statistical power. The effect of the country of origin can be observed when analyzing the results; for example, samples imported from the Netherlands were highly contaminated with Penicillium when compared to samples from other countries. On the other hand, Penicillium is known to be more common in citrus fruits. However, the data in Table-1 do not demonstrate a significant difference between the number of Penicillium isolated from oranges and those isolated from the rest of the fruits, which might link Penicillium to the country of origin.

The data indicate also high concentrations of Cladosporium in samples originating specifically from Morocco. Interestingly, a total of 16 samples of cucumber were collected from Iran (six samples), Qatar (six samples), and Lebanon (four samples). Out of the six locale samples, five were contaminated with Fusarium, suggesting that most Fusarium isolates are local in origin. Finally, it is worth mentioning that the rate of occurrence of Aspergillus and Rhizopus is particularly high in samples originated from the USA. However, this high rate might be more related to the fruits' nature than to the country of origin, as those two fungal types were linked to strawberry infections knowing that most of these fruit samples were of American origin (data not shown).

\section{Effect of the country of origin's economy on fruit} spoilage

The various countries of origin of the samples were divided into countries categorized as having a developing economy and those with a developed economy (as based on the Department of Economic and Social Affairs of the United Nations Secretariat). Chisquare test was again applied to determine whether a countries' economic classification affected the fungal contamination frequency of their fruits. Although the test returned a non-significant $\mathrm{p}=0.081$ and $>0.05$, Table- 2 indicates that $49 \%$ of the samples originating from developing economy countries were contaminated, while $67.6 \%$ of samples from developed economy countries showed fungal growth within 10 days. The statistical results are likely to be biased by the fact that different fruits were exported from specific countries; for example, strawberries are more prone 
to spoilage than other fruits and, due to the nature of their skin, tend to be produced in developed nations.

\section{Effect of price of commodity on spoilage rate}

The collected fruit samples ranged in price from items that were on promotion, to very expensive fruit types. Therefore, it is important to interrogate the effect of the price of fruit on their home shelf-life to understand if, in the market, low price reflects the microbiological condition of the commodity or if it is a reflection of the taste and/or probable variation in the nutritional value. The average price per commodity was calculated by SPSS, as shown in Table-3.

High and low price categories were created by considering fruits whose price was above or below the

Table-2: Number of samples originated from countries of different economy and their contamination frequency.

\begin{tabular}{lccc}
\hline Contamination & \multicolumn{2}{c}{ Economy } & Total \\
\cline { 2 - 3 } & Developed & Developing & \\
\hline No & 12 & 27 & 39 \\
Yes & 25 & 26 & 51 \\
Total & 37 & 53 & 90 \\
\hline
\end{tabular}

Table-3: Descriptive statistics of the prices QAR/kg of different fruit types.

\begin{tabular}{lccc}
\hline Sample type & Mean & $\mathbf{n}$ & Standard deviation \\
\hline Cucumber & 8.20 & 16 & 4.38 \\
Orange & 8.85 & 30 & 5.46 \\
Strawberry & 63.02 & 14 & 18.30 \\
Tomato & 16.31 & 30 & 6.81 \\
Total & 19.65 & 90 & 20.96 \\
\hline
\end{tabular}

average for their category respectively. Chi-square test was computed to assess the effect of price category on spoilage rate. Chi-square results are for cucumber $(p=0.267>0.05)$, orange $(p=0.232>0.05)$, and tomato $(\mathrm{p}=0.296>0.05)$ indicate a non-significant effect. The test could not be conducted on strawberries because all samples were contaminated. In the three categories of fruits tested, the data did not provide evidence that more expensive fruits are less contaminated or last longer. Instead, price variation may be a product of the quality of the commodity, rather than its home shelf-life.

\section{Number of fungal types per sample}

Some fruits were found to be contaminated with more than one, but no more than three, types of fungi at once. Data were summarized in a boxplot and the results indicate that strawberry samples contain the greatest variety of fungi of all the fruits. This range of contamination is due to the nature of the skin of this fruit, which contains pouches that fungal spores get stuck within. It should be noted that strawberries were primarily contaminated with black molds, whose spores can easily be transmitted by air. The cucumbers data demonstrate the existence of some outliers, but its median is at one fungal type per sample, whereas tomatoes showed high specificity in terms of fungal types and oranges did not have enough contaminated samples to be included (Figure-1).

The P-P plot of the number of fungal types indicates that the number of fungal types per fruit sample follows a normal distribution (data not shown). SPSS was, therefore, used to perform an ANOVA test to compare the mean numbers of contaminant kinds

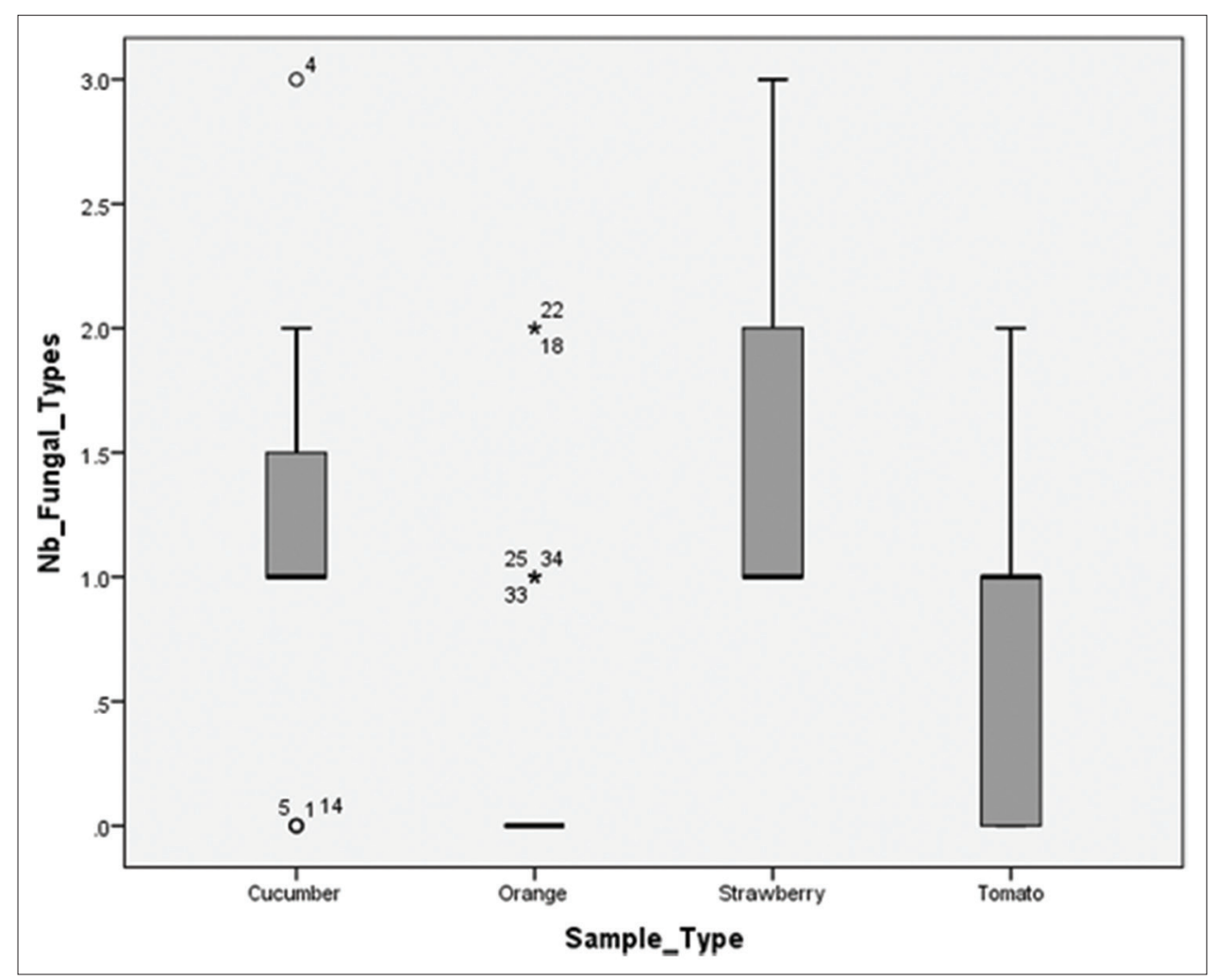

Figure-1: Box plot of the number of fungal contaminants by sample type. 
per sample type. The results $(p<0.01)$ suggest that fruits do significantly vary in the number of fungal types they carry. Post hoc tests show that the main significant differences in the number of fungi are between cucumbers and oranges, oranges and strawberries, tomatoes and oranges, and tomatoes and strawberries.

\section{Contamination level of various fruit samples}

The level of contamination across different fruits was assessed based on the number of spots shown on each sample at the beginning of the rotting process. Because each spot represents a spore, their number reflects the level of contamination. The levels were described as follows:

1. Undetectable contamination level: Zero spots (within 10 days)

2. Low contamination level: 1-4 spots

3. Moderate contamination level: 5-10 spots

4. Heavy contamination level: Sample fully covered with fungus

5. Detected by swab: Contamination was not visible, but proven by swab culture.
Figure-2 summarizes the contamination levels of each of the fruits. Strawberries exhibited the highest levels of fungi per fruit; again, this may result from the characteristics of its skin. Oranges exhibited a high level of contamination across $13.3 \%$ of samples, whereas more than half of the cucumber samples $(56.25 \%)$ showed no hyphae growth by the end of the 10 days at all. However, the visibly bad condition of the fruit motivated us to take a swab culture from them on PDA, all of which demonstrated the growth of various fungi types. Finally, it is worth mentioning that $60 \%$ of the tomato samples were contaminated but that most of those $(53.3 \%)$ exhibited only a low spread of contaminations per fruit, which can be explained by the smooth, thick nature of the tomatoes' skin.

\section{The home shelf-life of various fruits}

The time taken by a fruit sample to rot will vary from 1 to more than 10 days. Based on the rotting speed, samples were divided as follows:

1. Speed 1 samples: Very short home shelf-life, rotten between 1 and 3 days

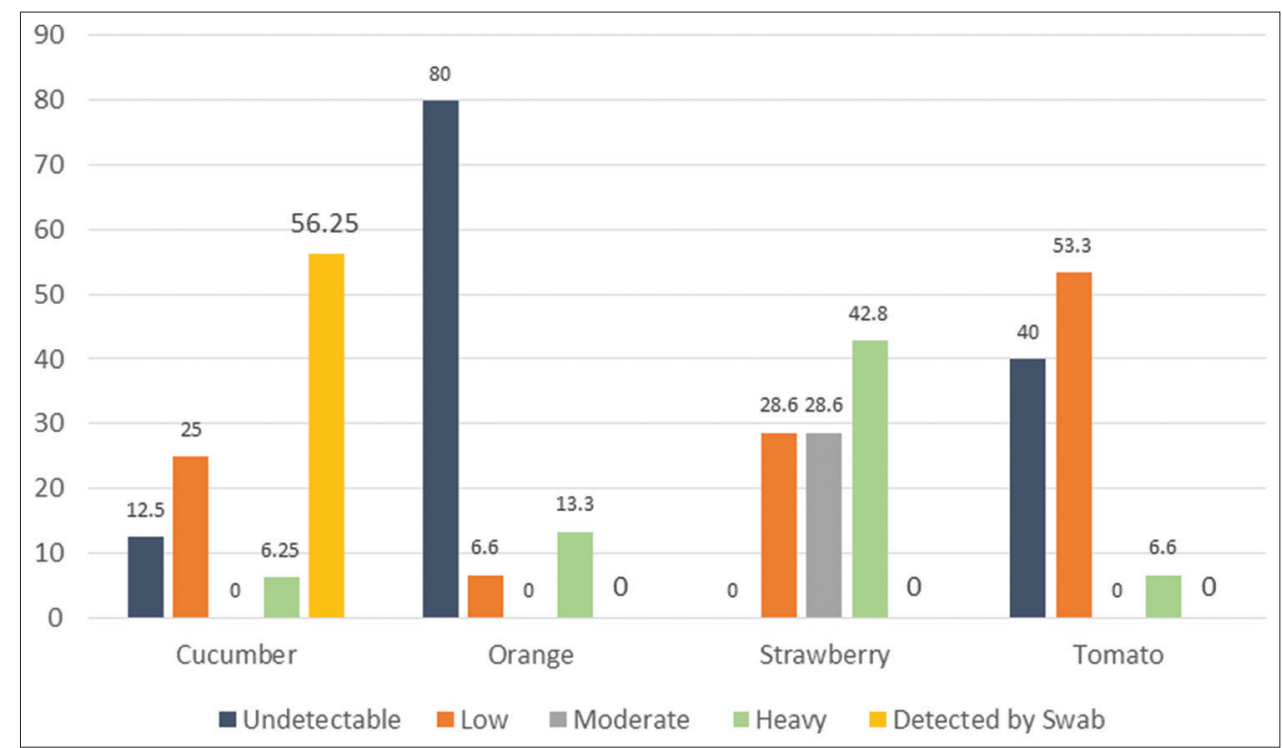

Figure-2: The percentage of various fruits that have undetected, low, moderate, heavy, and swab detected contamination levels.

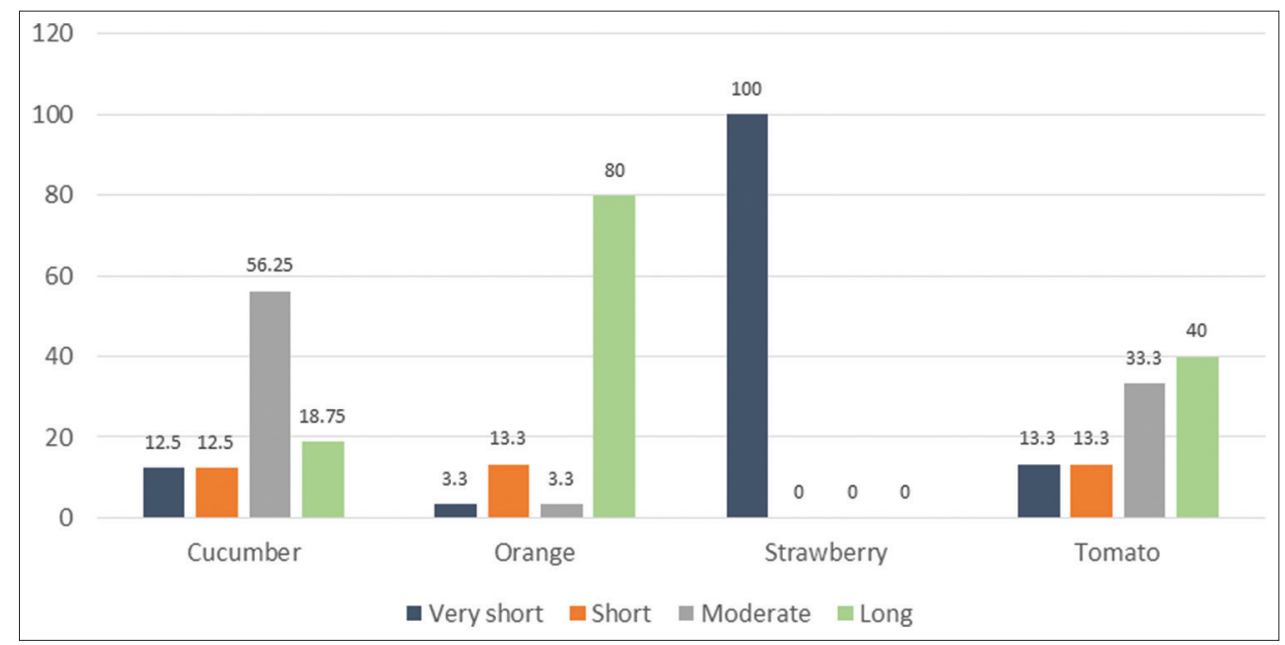

Figure-3: Percentages of various fruits that have very short, short, moderate, and long home shelf-life. 
2. Speed 2 samples: Short home shelf-life, rotten between 4 and 6 days

3. Speed 3 samples: Moderate home shelf-life, rotten between 7 and 10 days

4. Speed 4 samples: Long home shelf-life, the samples did not rot within the experiment time.

Strawberries experienced the shortest home shelflife, with all samples rotting within 3 days. This period was followed shortly by cucumbers, of which $87.5 \%$ of the samples were in a bad condition within 10 days. About $40 \%$ of the tomato samples and $80 \%$ of the orange samples remained in a good and appealing condition during the 10 days of the experiment (Figure-3).

\section{Discussion}

Fungi have always been a public health concern. In 1992, a study conducted in Australia on packaged cheddar cheese showed that mold infections were tough to control as they were isolated from different equipment in the cheese factory, fungi were also isolated from the air, curd, and whey. The most common fungi isolated from the packaged cheese samples were Cladosporium followed by Penicillium, which are our most commonly isolated fungi [14]. Similarly, in 2001, Weidenborner determined the major source of contamination for pine nuts as Cladosporium. A total of 31 different species were isolated in the study, of which 16 were potentially toxigenic [15].

Recent studies regarding fungal contamination are conducted all over the world. A total of 117 isolates of fungi were recovered from nuts and dried fruits in the Washington D.C. area, including potential toxigenic Aspergillus, Penicillium, Alternaria, and Fusarium species. Similarly, the most common isolated fungi type identified in a study by Tournas et al. [16] was Aspergillus, while the most common mold in walnuts was identified as Penicillium. In Brazil, a 2007 study investigating toxigenic fungi on dried fruits identified Aspergillus niger as the most common species from a sample of 117 [17]. Both studies imply an affinity between Aspergillus and dried fruits.

Similarly, a study of grapes conducted in Lebanon identified a high level of contamination with various strains of Aspergillus section Nigri. In addition, tests of ochratoxin A (OTA) levels indicated low-level contamination of the isolates in $57.4 \%$ of cases which, again, illustrates the risk to human health posed by the toxins produced by Aspergillus species, the third most isolated species in the present study [13].

Aflatoxin and OTA producing fungi are also of major concern in cocoa production. During the chocolate production process but, in particular, during the fermentation of the cocoa beans, molds contamination is common [18]. In a study conducted in Brazil, in 2014, out of 356 chocolate samples tested, 303 (85.1\%) showed traces of OTA. Although the detected toxin levels were within acceptable ranges, chocolate-based products are widely consumed, especially by children, and the accumulative effect of low-level OTA consumption may cause severe health problems in vulnerable populations, making the control of toxin-producing fungi of major importance [19].

While the orange samples considered in the present study displayed the highest level of contamination with Penicillium, the previous study conducted on sweet oranges in Nigeria found isolates of $A$. niger to be the most numerous (27.5\%) followed by Rhizopus (22.5\%) and Aspergillus flavus (17.5\%) [20]. In close consistency with our results, the second and third most common spoiling agents of our study were isolated in Uyo metropolis, Nigeria, from samples of cucumbers, carrots, cabbages, and onions. Approximately one-third or $37.5 \%$ of the Nigerian samples showed contamination with Rhizopus stolonifer and with Aspergillus fumigatus, while A. niger was found only in cucumber and onion samples, which constituted $25 \%$ of the overall number of samples [21]. Eight of our 11 Botrytis isolates were isolated from the strawberry sample, which is consistent with a study conducted in Virginia USA on strawberries, which later revealed that the most abundant fungi among the fruit were Botrytis and Cladosporium [22].

In the Gulf region, studies conducted on fungal contamination levels are few. A study conducted in Bahrain on 17 types of imported spices showed that black pepper and red chili have the highest fungal contamination. The most encountered fungal type was Aspergillus, which was isolated from four types of spices followed by Penicillium, which was isolated from two types. Cladosporium, Rhizopus, and Trichoderma were also frequently isolated [23]. In Saudi Arabia, 520 samples of date Rutab were tested for microbial spoilage. The two main spoilage-causing agents were Penicillium and Cladosporium [24]. Despite the various sample types, the results of the two studies are in harmony with our results.

This study illustrates the high level of fungi present in the food products that are consumed daily. However, there are many steps to take that can help avoid human exposure to such microorganisms and conserve imported fruits and vegetables for longer periods of time. Among the required steps are the implementation of programs such as hazard analysis and critical control point (HACCP) in places where food is processed and produced. Programs such as HACCP help to establish the critical control points used to monitor food quality continuously and they help to provide appropriate training to food handlers at all food production stages. Although such measures are costly, they are necessary to ensure appropriate food quality control $[25,26]$.

\section{Conclusion}

Food spoilage monitoring and spoiling agents' identification is crucial steps in food management programs. Food security is a global concern in both developed and developing countries. The world population has increased from 1.5 to 6.9 billion between 1900 and 2000. This booming comes with an increase 
in food demand. Fresh fruits and vegetables microbial spoilage causes huge economic losses every year in Qatar and all around the world. Development of spoilage control methods requires first a good knowledge of existing spoiling agents in each country and about the factors that control their growth. It is clear in this analysis that the displaying practices and the prices of the commodities are not food spoilage affecting factors in Qatar, rather fresh produces country of origin and locally produced crops require further attention.

\section{Authors' Contributions}

IS and RA conceived and designed the experiments and wrote the manuscript. IS performed the experiments. Both authors read and approved the final manuscript.

\section{Acknowledgments}

The authors wish to thank Dr. Mohammed AbuDieyeh at the Biological and Environmental Sciences Department at Qatar University for his assistance in identifying some of the isolated species and Mr. Ahmad Hawi for his valuable input in the statistical analysis. This study was partially funded by Biological and Environmental Science Department of Qatar University.

\section{Competing Interests} interests.

The authors declare that they have no competing

\section{Publisher's Note}

Veterinary World remains neutral with regard to jurisdictional claims in published institutional affiliation.

\section{References}

1. WHO. Available from: http://www.who.int/features/factfiles/food_safety/en.2016. Last accessed on 12-09-2019.

2. Aguiar, R.S., Esmerino, E.A., Rocha, R.S., Pimentel, T.C., Alvarenga, V.O., Freitas, M.Q., Silva, M.C., Sant'Ana, A.S., Silva, A.C.O. and Cruz, A.G. (2018) Physical hazards in dairy products: Incidence in a consumer complaint website in Brazil. Food Control, 86: 66-70.

3. Snyder, A.B. and Worobo, R.W. (2018) The incidence and impact of microbial spoilage in the production of fruit and vegetable juices as reported by juice manufacturers. Food Control, 85: 144-150.

4. Giampieri, F., Tulipani, S., Alvarez-Suarez, J.M., Quiles, J.L., Mezzetti, B. and Battino, M. (2012) The strawberry: Composition, nutritional quality, and impact on human health. Nutrition, 28(1): 9-19.

5. Mailafia, S., Okoh, G.R., Olabode, H.O.K. and Osanupin, R (2017) Isolation and identification of fungi associated with spoilt fruits vended in Gwagwalada market, Abuja, Nigeria. Vet. World, 10(4): 393-397.

6. Tournas, V.H. (2005) Spoilage of vegetable crops by bacteria and fungi and related health hazards. Crit. Rev. Microbiol., 31(1): 33-44.

7. Moss, M.O. (2008) Fungi, quality and safety issues in fresh fruits and vegetables. J. Appl. Microbiol., 104(5): 1239-1243.

8. Perrone, G. and Gallo, A. (2017) Aspergillus species and their associated mycotoxins. Methods Mol. Biol., 1542: 33-49.

9. Pinto, V.E. and Patriarca, A. (2017) Alternaria species and their associated mycotoxins. Methods Mol. Biol., 1542: 13-32.

10. Gomba, A., Chidamba, L. and Korsten, L. (2017) Effect of postharvest practices including degreening on citrus carpoplane microbial biomes. J. Appl. Microbiol., 122(4): 1057-1070.

11. Mehyar, G.F., Al-Qadiri, H.M., Abu-Blan, H.A. and Swanson, B.G. (2011) Antifungal effectiveness of potassium sorbate incorporated in edible coatings against spoilage molds of apples, cucumbers, and tomatoes during refrigerated storage. J. Food Sci., 76(3): M210-M217.

12. Ham, H., Kim, S., Kim, M.H., Lee, S., Hong, S.K., Ryu, J.G. and Lee, T. (2016) Mycobiota of ground red pepper and their aflatoxigenic potential. J. Microbiol., 54(12): 832-837.

13. El Khoury, A., Rizk, T., Lteif, R., Azouri, H., Delia, M.L. and Lebrihi, A. (2006) Occurrence of ochratoxin A and aflatoxin B1-producing fungi in Lebanese grapes and ochratoxin A content in musts and finished wines during 2004. $J$. Agric. Food Chem. 54(23): 8977-8982.

14. Hocking, A.D. and Faedo, M. (1992) Fungi causing thread mould spoilage of vacuum packaged cheddar cheese during maturation. Int. J. Food Microbiol., 16(2): 123-130.

15. Weidenborner, M. (2001) Pine nuts: The mycobiota and potential mycotoxins. Can. J. Microbiol., 47(5): 460-463.

16. Tournas, V.H., Niazi, N.S. and Kohn, J.S. (2015) Fungal presence in selected tree nuts and dried fruits. Microbiol. Insights, 8: 1-6.

17. Iamanaka, B.T., Taniwaki, M.H., Menezes, H.C., Vicente, E. and Fungaro, M.H. (2005) Incidence of toxigenic fungi and ochratoxin A in dried fruits sold in Brazil. Food Addit. Contam., 22(12): 1258-1263.

18. Kutsanedzie, F.Y.H., Chen, Q., Hassan, M.M., Yang, M., Sun, H. and Rahman, M.H. (2018) Near infrared system coupled chemometric algorithms for enumeration of total fungi count in cocoa beans neat solution. Food Chem., 240: 231-238.

19. Copetti, M.V., Iamanaka, B.T., Pitt, J.I. and Taniwaki, M.H. (2014) Fungi and mycotoxins in cocoa: From farm to chocolate. Int. J. Food Microbiol., 178: 13-20.

20. Samuel, O., Ifeanyi, O. and Ugochukwu, O. (2015) Filamentous fungi associated with the spoilage of post-harvest sweet orange fruits (Citrus sinensis) sold in Awka major markets, Nigeria. Bioeng. Biosci., 3(3): 44-49.

21. Adebayo-Tayo, B., Odu, N., Cu, E. and Okonko, I. (2012) Microorganisms associated with spoilage of stored vegetables in Uyo metropolis, Akwa Ibom state, Nigeria. Nat. Sci., 10(3): 23-32

22. Abdelfattah, A., Wisniewski, M., Li Destri Nicosia, M.G., Cacciola, S.O. and Schena, L. (2016) Metagenomic analysis of fungal diversity on strawberry plants and the effect of management practices on the fungal community structure of aerial organs. PLoS One, 11(8): e0160470.

23. Mandeel, Q.A. (2005) Fungal contamination of some imported spices. Mycopathologia, 159(2): 291-298.

24. Hamad, S.H. (2008) Microbial spoilage of date rutab collected from the markets of Al-Hofuf city in the kingdom of Saudi Arabia. J. Food Prot., 71(7): 1406-1411.

25. Cusato, S., Garneiro, A.H., Sant'Ana, A., Corassin, C., Cruz, A. and Oliveira, C. (2014) Assessing the costs involved in the implementation of GMP and HACCP in a small dairy factory. Qual. Assur. Saf. Crops Foods, 6(2): 135-139.

26. Gomes, C.C.B., Lemos, G.F.C., Silva, M.C., Hora, I.M.C. and Cruz, A. (2014) Training of food handlers in a hotel: Tool for promotion of the food safety. J. Food Saf., 34(3): 218-223. 